\title{
A Frustrated Lewis Pair Based on a Cationic Aluminium Complex and Triphenylphosphine
}

\author{
Tom E. Stennett, Jürgen Pahl, Harmen S. Zijlstra, Falk W. Seidel and Sjoerd Harder* \\ Inorganic and Organometallic Chemistry, Friedrich-Alexander-Universität Erlangen-Nürnberg, Egerlandstraße 1, \\ 91058 Erlangen, Germany.
}

\section{Contents}

Crystal Structure Determination.....

Figure S1 - Crystal structure of compound 2

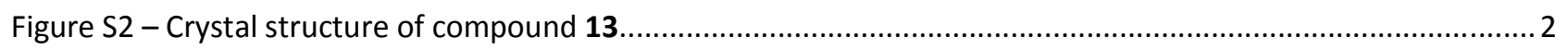

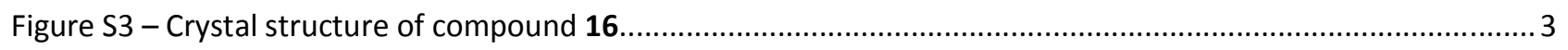

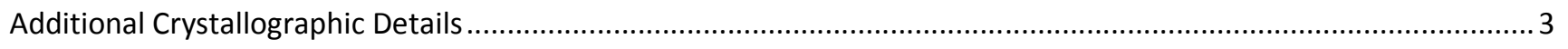

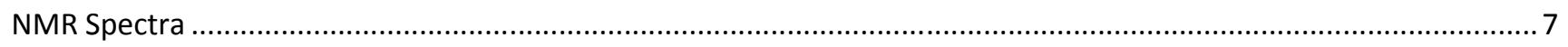

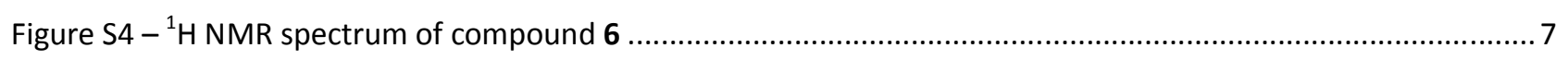

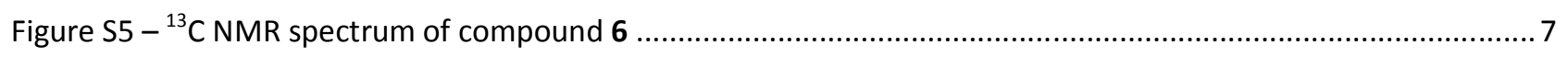

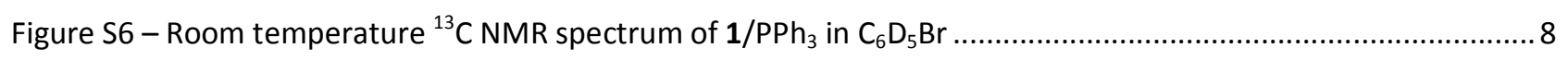

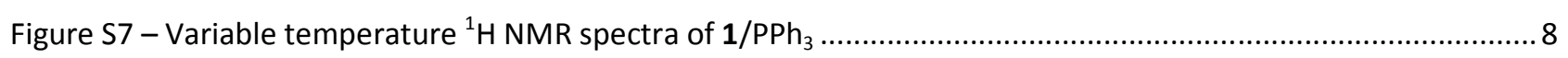

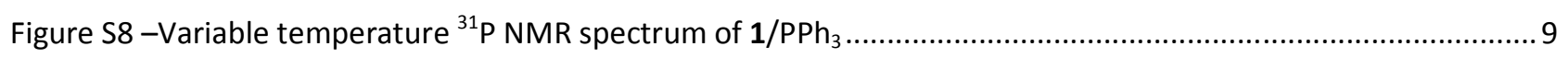

Figure S9-Standard ${ }^{1} \mathrm{H}$ NMR spectrum (below) and selective TOCSY ${ }^{1} \mathrm{H}$ NMR spectrum (above) of compound $\mathbf{9}$,

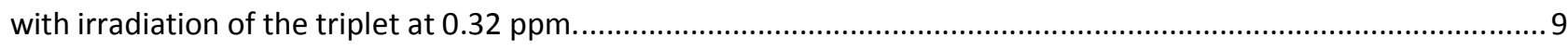

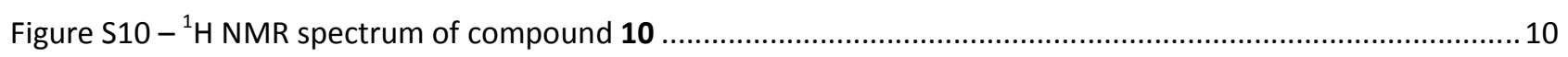

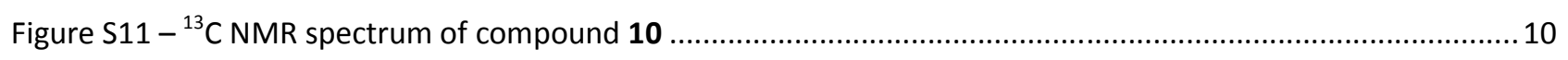

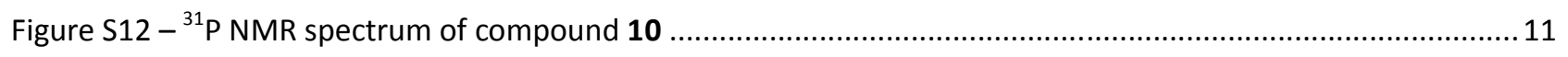

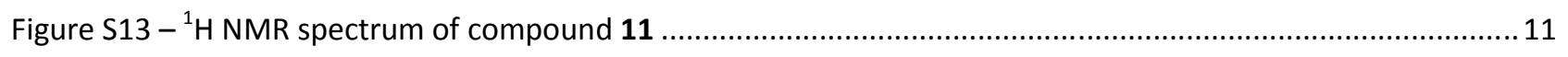

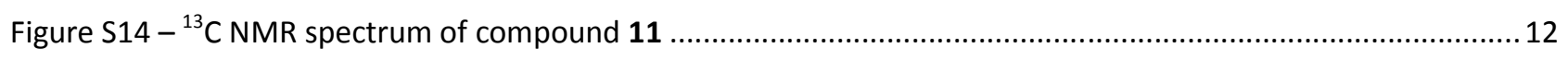

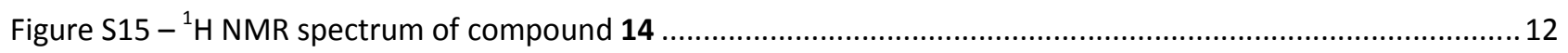

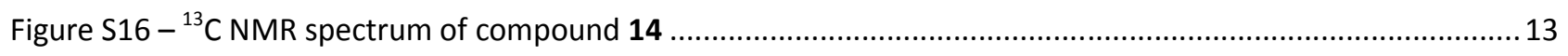

Figure S17 - ${ }^{31}$ P NMR spectra for the Gutmann-Beckett Lewis acidity test ......................................................... 13 


\section{Crystal Structure Determination}

Figure S1 - Crystal structure of compound 2. Hydrogen atoms, ${ }^{i}$ Pr groups and the $\left[B\left(\mathrm{C}_{6} \mathrm{~F}_{5}\right)_{4}\right]^{-}$anion are omitted for clarity.

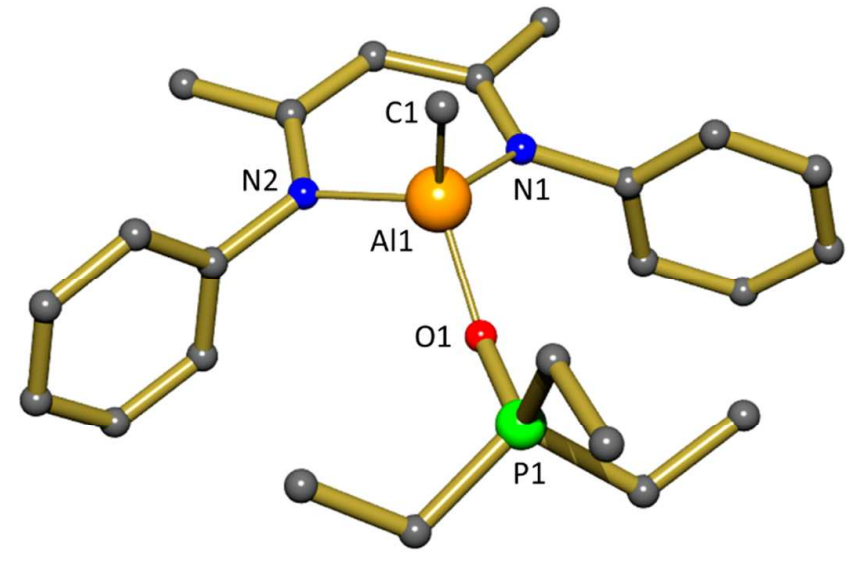

Figure S2 - Crystal structure of compound 13. Hydrogen atoms, ${ }^{\prime} \mathrm{Pr}$ groups and the $\left[\mathrm{B}\left(\mathrm{C}_{6} \mathrm{~F}_{5}\right)_{4}\right]^{-}$anion are omitted for clarity.

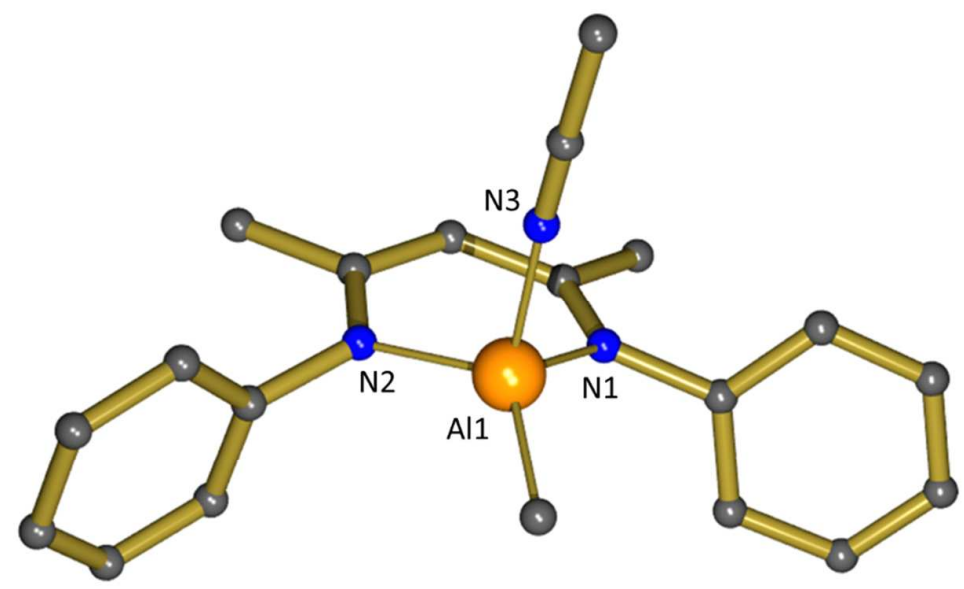


Figure S3 - Crystal structure of compound 16. Hydrogen atoms except for $\mathrm{H} 1 \mathrm{a}$ and $\mathrm{H} 1 \mathrm{~b}$ are omitted for clarity.

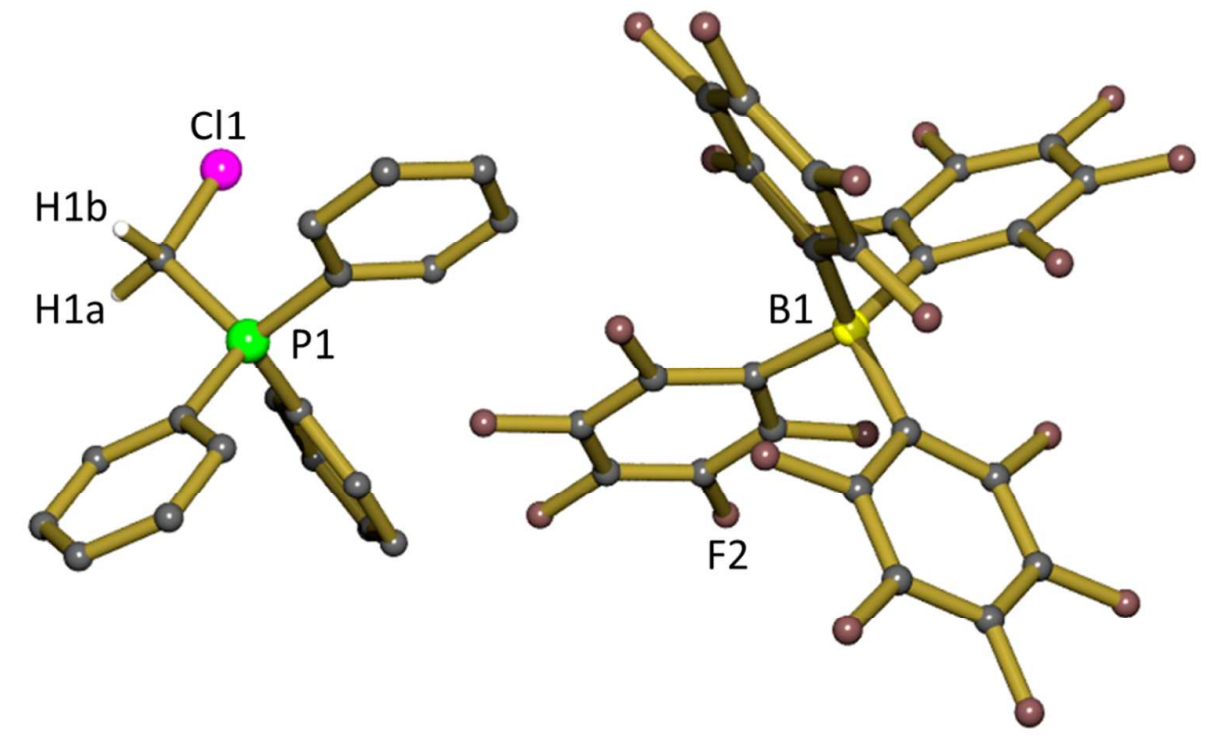

\section{Additional Crystallographic Details}

\section{Compound 2:}

The solvent molecule was modeled with a Rigid Body (RIGU) Restraint. The co-crystallised solvent was split in two positions of benzene/bromobenzene in a ratio 95:5.

Compound 6:

The observed solvent accessible voids $\left(189 \AA^{3}\right)$ contain disordered solvent. A satisfactory disorder model for the solvent was not found, and therefore the OLEX2 Solvent Mask routine (similar to PLATON/SQUEEZE) was used to mask out the disordered electron density (50e-) [1].

\section{Compound 9:}

The observed solvent accessible voids (196 $\AA^{3}$ per asymmetric unit) contain disordered solvent. A satisfactory disorder model for the solvent was not found, and therefore the OLEX2 Solvent Mask routine (similar to PLATON/SQUEEZE) was used to mask out the disordered electron density (50e per asymmetric unit). Two pentafluorophenyl substituents are split over two disordered positions each with an approximate ratio of 70:30. The less occupied part of each pentafluorophenyl substituent was modeled with an idealized geometry (AFIX 66) and Rigid Bond (RIGU) Restraints.

\section{Compound 10:}

The observed solvent accessible voids $\left(300 \AA^{3}\right)$ contain disordered solvent. A satisfactory disorder model for the solvent was not found, and therefore the OLEX2 Solvent Mask routine (similar to PLATON/SQUEEZE) was used to mask out the disordered electron density (50e-) [1].

\section{Compound 13:}

The observed solvent accessible voids $\left(155 \AA^{3}\right)$ contain disordered solvent. A satisfactory disorder model for the solvent was not found, and therefore the OLEX2 Solvent Mask routine (similar to PLATON/SQUEEZE) was used to mask out the disordered electron density (50e-) [1].

[1] Acta Cryst. D61, 2005, 1299-1301 


\begin{tabular}{|c|c|c|c|}
\hline Identification code & Compound 2 & Compound 6 & Compound 7 \\
\hline Empirical formula & $\mathrm{C}_{66} \mathrm{H}_{64.95} \mathrm{AlBBr}_{0.05} \mathrm{~F}_{20} \mathrm{~N}_{2} \mathrm{OP}$ & $\mathrm{C}_{62} \mathrm{H}_{50} \mathrm{AlBF}_{20} \mathrm{~N}_{2}$ & $\mathrm{C}_{72} \mathrm{H}_{59} \mathrm{AlBF}_{20} \mathrm{~N}_{2} \mathrm{P}$ \\
\hline Formula weight & 1354.90 & 1240.83 & 1400.97 \\
\hline Temperature/K & 100 & 100 & 100 \\
\hline Crystal system & monoclinic & triclinic & triclinic \\
\hline Space group & $\mathrm{P} 2_{1} / \mathrm{n}$ & $\mathrm{P}-1$ & $\mathrm{P}-1$ \\
\hline $\mathrm{a} / \AA ̊$ & $12.88268(18)$ & $11.4727(4)$ & $12.6665(3)$ \\
\hline $\mathrm{b} / \AA$ & $15.0778(3)$ & $16.3099(4)$ & $14.7992(4)$ \\
\hline$c / \AA$ & $33.4651(5)$ & $16.4907(5)$ & $18.4930(5)$ \\
\hline$\alpha /^{\circ}$ & 90 & $93.934(2)$ & $76.039(2)$ \\
\hline$\beta /^{\circ}$ & $99.3265(15)$ & $107.911(3)$ & $78.861(2)$ \\
\hline $\mathrm{V} /{ }^{\circ}$ & 90 & $91.817(2)$ & $89.216(2)$ \\
\hline Volume $/ \AA^{3}$ & $6414.41(17)$ & 2924.71(16) & $3298.82(16)$ \\
\hline Z & 4 & 2 & 2 \\
\hline$\rho_{\text {calc }} g / \mathrm{cm}^{3}$ & 1.403 & 1.409 & 1.410 \\
\hline$\mu / \mathrm{mm}^{-1}$ & 1.454 & 1.243 & 1.392 \\
\hline$F(000)$ & 2791.0 & 1268.0 & 1436.0 \\
\hline Crystal size $/ \mathrm{mm}^{3}$ & $\begin{array}{l}0.2208 \times 0.1353 \times \\
0.0722\end{array}$ & $\begin{array}{l}0.3152 \times 0.1529 \times \\
0.0952\end{array}$ & $\begin{array}{l}0.1741 \times 0.1325 \times \\
0.0826\end{array}$ \\
\hline Crystal colour & colourless & colourless & colourless \\
\hline Radiation & CuK $\alpha(\lambda=1.54184)$ & $\operatorname{CuK\alpha }(\lambda=1.54184)$ & CuK $\alpha(\lambda=1.54184)$ \\
\hline $2 \Theta$ range for data collection $/^{\circ}$ & 7.846 to 136.228 & 7.516 to 147.374 & 6.158 to 136.184 \\
\hline Index ranges & $\begin{array}{l}-12 \leq h \leq 15,-15 \leq k \leq 18 \\
-40 \leq l \leq 40\end{array}$ & $\begin{array}{l}-14 \leq \mathrm{h} \leq 13,-15 \leq \mathrm{k} \leq \\
20,-20 \leq \mathrm{I} \leq 19\end{array}$ & $\begin{array}{l}-15 \leq \mathrm{h} \leq 15,-17 \leq \mathrm{k} \leq \\
12,-22 \leq \mathrm{l} \leq 21\end{array}$ \\
\hline Reflections collected & 21703 & 18288 & 20392 \\
\hline Independent reflections & $\begin{array}{l}11649\left[R_{\text {int }}=0.0328,\right. \\
\left.R_{\text {sigma }}=0.0420\right]\end{array}$ & $\begin{array}{l}11268\left[R_{\text {int }}=0.0257,\right. \\
\left.R_{\text {sigma }}=0.0374\right]\end{array}$ & $\begin{array}{l}11948\left[R_{\text {int }}=0.0257,\right. \\
\left.R_{\text {sigma }}=0.0362\right]\end{array}$ \\
\hline Data/restraints/parameters & $11649 / 36 / 852$ & $11268 / 0 / 786$ & $11948 / 0 / 885$ \\
\hline Goodness-of-fit on $\mathrm{F}^{2}$ & 1.017 & 1.022 & 1.022 \\
\hline Final $R$ indexes $[I>=2 \sigma(I)]$ & $\begin{array}{l}R_{1}=0.0373, w R_{2}= \\
0.0946\end{array}$ & $\begin{array}{l}R_{1}=0.0353, w R_{2}= \\
0.0891\end{array}$ & $\begin{array}{l}R_{1}=0.0337, w R_{2}= \\
0.0850\end{array}$ \\
\hline Final $\mathrm{R}$ indexes [all data] & $\begin{array}{l}R_{1}=0.0429, w R_{2}= \\
0.0994\end{array}$ & $\begin{array}{l}\mathrm{R}_{1}=0.0410, \mathrm{wR}_{2}= \\
0.0935\end{array}$ & $\begin{array}{l}\mathrm{R}_{1}=0.0390, \mathrm{wR}_{2}= \\
0.0899\end{array}$ \\
\hline Largest diff. peak/hole / e $\AA^{-3}$ & $0.51 /-0.27$ & $0.31 /-0.27$ & $0.38 /-0.36$ \\
\hline
\end{tabular}




\begin{tabular}{|c|c|c|c|}
\hline Identification code & Compound 8 & Compound 9 & Compound 10 \\
\hline Empirical formula & $\mathrm{C}_{80} \mathrm{H}_{65} \mathrm{AlBF}_{20} \mathrm{~N}_{2} \mathrm{P}$ & $\mathrm{C}_{78} \mathrm{H}_{71} \mathrm{AlBF}_{20} \mathrm{~N}_{2} \mathrm{P}$ & $\mathrm{C}_{73} \mathrm{H}_{59} \mathrm{AlBF}_{20} \mathrm{~N}_{2} \mathrm{O}_{2} \mathrm{P}$ \\
\hline Formula weight & 1503.10 & 1485.12 & 1444.98 \\
\hline Temperature/K & $100.01(10)$ & 100 & $100.01(10)$ \\
\hline Crystal system & triclinic & monoclinic & triclinic \\
\hline Space group & $\mathrm{P}-1$ & $\mathrm{P} 2_{1} / \mathrm{n}$ & $\mathrm{P}-1$ \\
\hline$a / \AA ̊$ & $11.0872(3)$ & $12.0147(3)$ & $12.0112(3)$ \\
\hline $\mathrm{b} / \AA ̊$ & $17.0047(4)$ & $31.3266(7)$ & $15.6917(4)$ \\
\hline$c / \AA$ & $19.8107(7)$ & $20.4013(4)$ & 19.6252(5) \\
\hline$\alpha /^{\circ}$ & $93.536(2)$ & 90 & $72.151(3)$ \\
\hline$\beta /^{\circ}$ & $105.636(3)$ & $92.019(2)$ & $88.283(2)$ \\
\hline $\mathrm{V} /{ }^{\circ}$ & $91.5752(18)$ & 90 & $86.427(2)$ \\
\hline Volume $/ \AA^{3}$ & $3586.17(17)$ & $7673.9(3)$ & $3513.76(17)$ \\
\hline Z & 2 & 4 & 2 \\
\hline$\rho_{\text {calcg }} g / \mathrm{cm}^{3}$ & 1.392 & 1.285 & 1.366 \\
\hline$\mu / \mathrm{mm}^{-1}$ & 1.321 & 1.225 & 1.347 \\
\hline$F(000)$ & 1544.0 & 3064.0 & 1480.0 \\
\hline Crystal size $/ \mathrm{mm}^{3}$ & $\begin{array}{l}0.2216 \times 0.1397 \times \\
0.1083\end{array}$ & $\begin{array}{l}0.3439 \times 0.2465 \times \\
0.1604\end{array}$ & $\begin{array}{l}0.2951 \times 0.248 \times \\
0.1775\end{array}$ \\
\hline Crystal colour & colourless & colourless & colourless \\
\hline Radiation & $\operatorname{CuK} \alpha(\lambda=1.54184)$ & CuK $\alpha(\lambda=1.54184)$ & CuK $\alpha(\lambda=1.54184)$ \\
\hline $2 \Theta$ range for data collection $/{ }^{\circ}$ & 6.73 to 136.224 & 7.116 to 136.22 & 6.356 to 147.176 \\
\hline Index ranges & $\begin{array}{l}-13 \leq \mathrm{h} \leq 9,-20 \leq \mathrm{k} \leq \\
20,-22 \leq \mathrm{I} \leq 23\end{array}$ & $\begin{array}{l}? \leq \mathrm{h} \leq ?, ? \leq \mathrm{k} \leq ?, ? \leq \mathrm{I} \\
\leq ?\end{array}$ & $\begin{array}{l}-14 \leq \mathrm{h} \leq 12,-16 \leq \mathrm{k} \leq \\
19,-24 \leq \mathrm{I} \leq 23\end{array}$ \\
\hline Reflections collected & 21128 & 13988 & 22235 \\
\hline Independent reflections & $\begin{array}{l}12990\left[R_{\text {int }}=0.0244,\right. \\
\left.R_{\text {sigma }}=0.0353\right]\end{array}$ & $\begin{array}{l}13988\left[R_{\text {int }}=?, R_{\text {sigma }}=\right. \\
0.0445]\end{array}$ & $\begin{array}{l}13554\left[R_{\text {int }}=0.0214\right. \\
\left.R_{\text {sigma }}=0.0307\right]\end{array}$ \\
\hline Data/restraints/parameters & $12990 / 0 / 957$ & $13988 / 211 / 1107$ & $13554 / 0 / 912$ \\
\hline Goodness-of-fit on $F^{2}$ & 1.016 & 1.073 & 1.047 \\
\hline Final $R$ indexes $[I>=2 \sigma(I)]$ & $\begin{array}{l}\mathrm{R}_{1}=0.0339, \mathrm{wR}_{2}= \\
0.0847\end{array}$ & $\begin{array}{l}R_{1}=0.0877, w R_{2}= \\
0.1957\end{array}$ & $\begin{array}{l}\mathrm{R}_{1}=0.0370, \mathrm{wR}_{2}= \\
0.0968\end{array}$ \\
\hline Final $R$ indexes [all data] & $\begin{array}{l}R_{1}=0.0381, w R_{2}= \\
0.0881\end{array}$ & $\begin{array}{l}R_{1}=0.0961, w R_{2}= \\
0.2008\end{array}$ & $\begin{array}{l}R_{1}=0.0409, w R_{2}= \\
0.0996\end{array}$ \\
\hline Largest diff. peak/hole / e $\AA^{-3}$ & $0.39 /-0.35$ & $0.97 /-0.63$ & $0.56 /-0.37$ \\
\hline
\end{tabular}




\begin{tabular}{|c|c|c|c|}
\hline Identification code & Compound 11 & Compound 13 & Compound 16 \\
\hline Empirical formula & $\mathrm{C}_{85} \mathrm{H}_{89} \mathrm{Al}_{2} \mathrm{BF}_{20} \mathrm{~N}_{4} \mathrm{O}_{2}$ & $\mathrm{C}_{56} \mathrm{H}_{47} \mathrm{AlBF}_{20} \mathrm{~N}_{3}$ & $\mathrm{C}_{43} \mathrm{H}_{17} \mathrm{BClF}_{20} \mathrm{P}$ \\
\hline Formula weight & 1643.37 & 1179.75 & 990.79 \\
\hline Temperature/K & 100 & $99.94(13)$ & 100 \\
\hline Crystal system & triclinic & monoclinic & monoclinic \\
\hline Space group & $\mathrm{P}-1$ & $\mathrm{P} 2_{1} / \mathrm{c}$ & $\mathrm{P} 2_{1} / \mathrm{n}$ \\
\hline$a / \AA ̊$ & $14.6101(2)$ & $17.2760(3)$ & $13.29789(9)$ \\
\hline $\mathrm{b} / \AA ̊$ & $16.7664(3)$ & $15.8452(2)$ & $30.4115(2)$ \\
\hline$c / \AA$ & $18.2804(4)$ & $20.1635(3)$ & $19.62736(14)$ \\
\hline$\alpha /^{\circ}$ & $91.2573(15)$ & 90 & 90 \\
\hline$\beta /^{\circ}$ & $104.8642(16)$ & $97.3436(15)$ & $104.3776(8)$ \\
\hline $\mathrm{V} /{ }^{\circ}$ & $105.6814(16)$ & 90 & 90 \\
\hline Volume $/ \AA^{3}$ & $4146.89(14)$ & $5474.33(14)$ & $7688.88(10)$ \\
\hline Z & 2 & 4 & 8 \\
\hline$\rho_{\text {calcg }} g / \mathrm{cm}^{3}$ & 1.316 & 1.431 & 1.712 \\
\hline$\mu / \mathrm{mm}^{-1}$ & 1.131 & 1.301 & 2.497 \\
\hline$F(000)$ & 1708.0 & 2408.0 & 3936.0 \\
\hline Crystal size $/ \mathrm{mm}^{3}$ & $\begin{array}{l}0.2076 \times 0.1626 \times \\
0.0955\end{array}$ & $\begin{array}{l}0.2325 \times 0.1388 \times \\
0.0713\end{array}$ & $\begin{array}{l}0.1985 \times 0.1563 \times \\
0.1135\end{array}$ \\
\hline Crystal colour & colourless & colourless & colourless \\
\hline Radiation & $\operatorname{CuK} \alpha(\lambda=1.54184)$ & CuK $\alpha(\lambda=1.54184)$ & $\operatorname{CuK} \alpha(\lambda=1.54184)$ \\
\hline $2 \Theta$ range for data collection $/{ }^{\circ}$ & 6.532 to 136.23 & 7.118 to 136.23 & 5.812 to 136.23 \\
\hline Index ranges & $\begin{array}{l}-17 \leq \mathrm{h} \leq 12,-19 \leq \mathrm{k} \leq \\
20,-19 \leq \mathrm{I} \leq 21\end{array}$ & $\begin{array}{l}-18 \leq \mathrm{h} \leq 20,-16 \leq \mathrm{k} \leq \\
19,-24 \leq \mathrm{I} \leq 15\end{array}$ & $\begin{array}{l}-16 \leq \mathrm{h} \leq 15,-36 \leq \mathrm{k} \leq \\
36,-14 \leq \mathrm{I} \leq 23\end{array}$ \\
\hline Reflections collected & 37200 & 17280 & 26941 \\
\hline Independent reflections & $\begin{array}{l}15030\left[R_{\text {int }}=0.0322,\right. \\
\left.R_{\text {sigma }}=0.0316\right]\end{array}$ & $\begin{array}{l}9827\left[R_{\text {int }}=0.0286,\right. \\
\left.R_{\text {sigma }}=0.0409\right]\end{array}$ & $\begin{array}{l}13897\left[R_{\text {int }}=0.0216,\right. \\
\left.R_{\text {sigma }}=0.0281\right]\end{array}$ \\
\hline Data/restraints/parameters & $15030 / 0 / 1049$ & $9827 / 0 / 742$ & $13897 / 0 / 1189$ \\
\hline Goodness-of-fit on $F^{2}$ & 1.024 & 1.029 & 1.047 \\
\hline Final $R$ indexes $[I>=2 \sigma(I)]$ & $\begin{array}{l}\mathrm{R}_{1}=0.0413, w \mathrm{R}_{2}= \\
0.1095\end{array}$ & $\begin{array}{l}R_{1}=0.0359, w R_{2}= \\
0.0878\end{array}$ & $\begin{array}{l}\mathrm{R}_{1}=0.0311, \mathrm{wR}_{2}= \\
0.0797\end{array}$ \\
\hline Final $R$ indexes [all data] & $\begin{array}{l}R_{1}=0.0455, w R_{2}= \\
0.1141\end{array}$ & $\begin{array}{l}R_{1}=0.0431, w R_{2}= \\
0.0933\end{array}$ & $\begin{array}{l}R_{1}=0.0341, w R_{2}= \\
0.0821\end{array}$ \\
\hline Largest diff. peak/hole / e $\AA^{-3}$ & $0.55 /-0.28$ & $0.41 /-0.28$ & $0.38 /-0.48$ \\
\hline
\end{tabular}


NMR Spectra

Figure $S 4-{ }^{1} \mathrm{H}$ NMR spectrum of compound 6

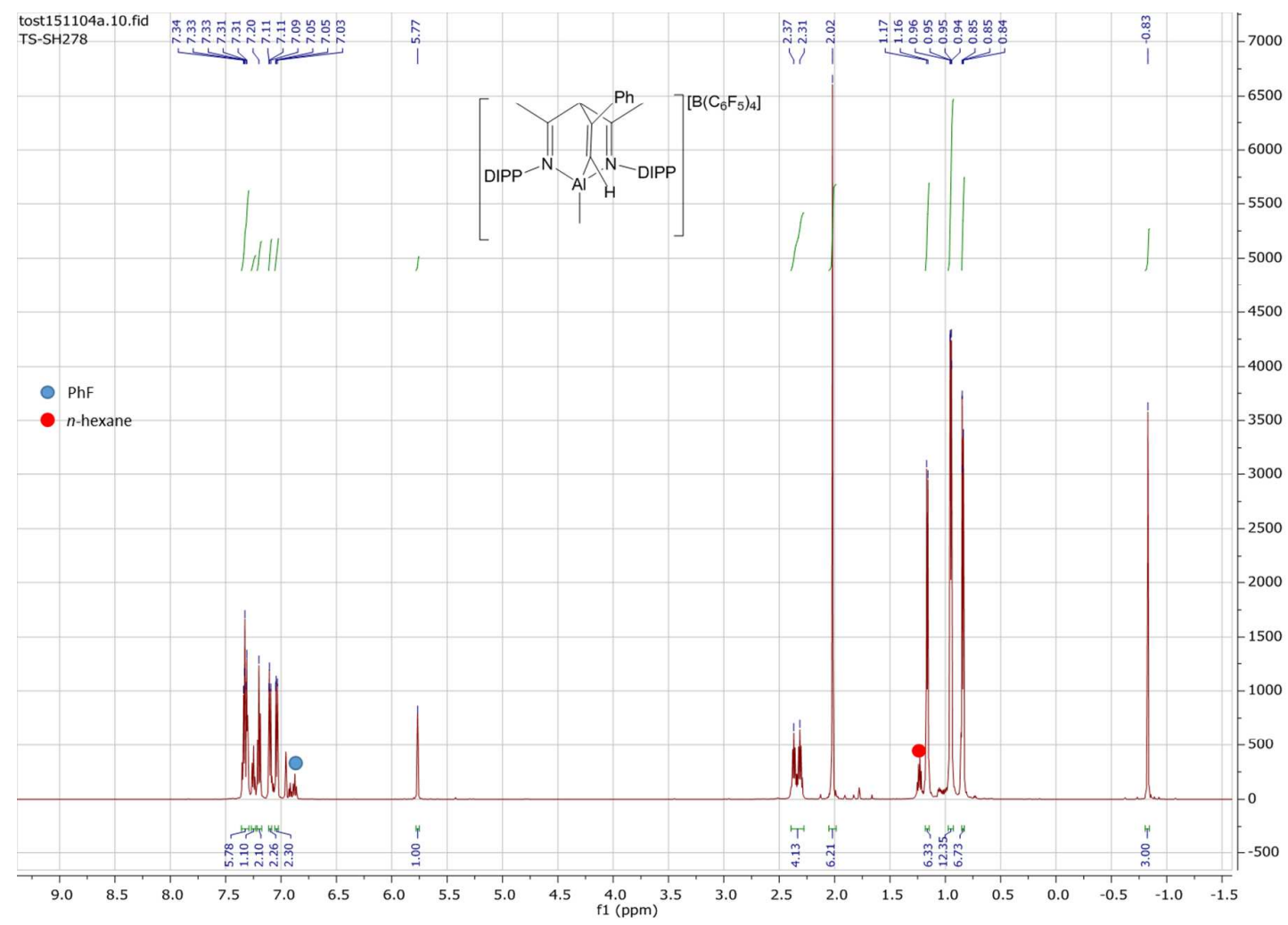

Figure S5 $-{ }^{13} \mathrm{C}$ NMR spectrum of compound 6

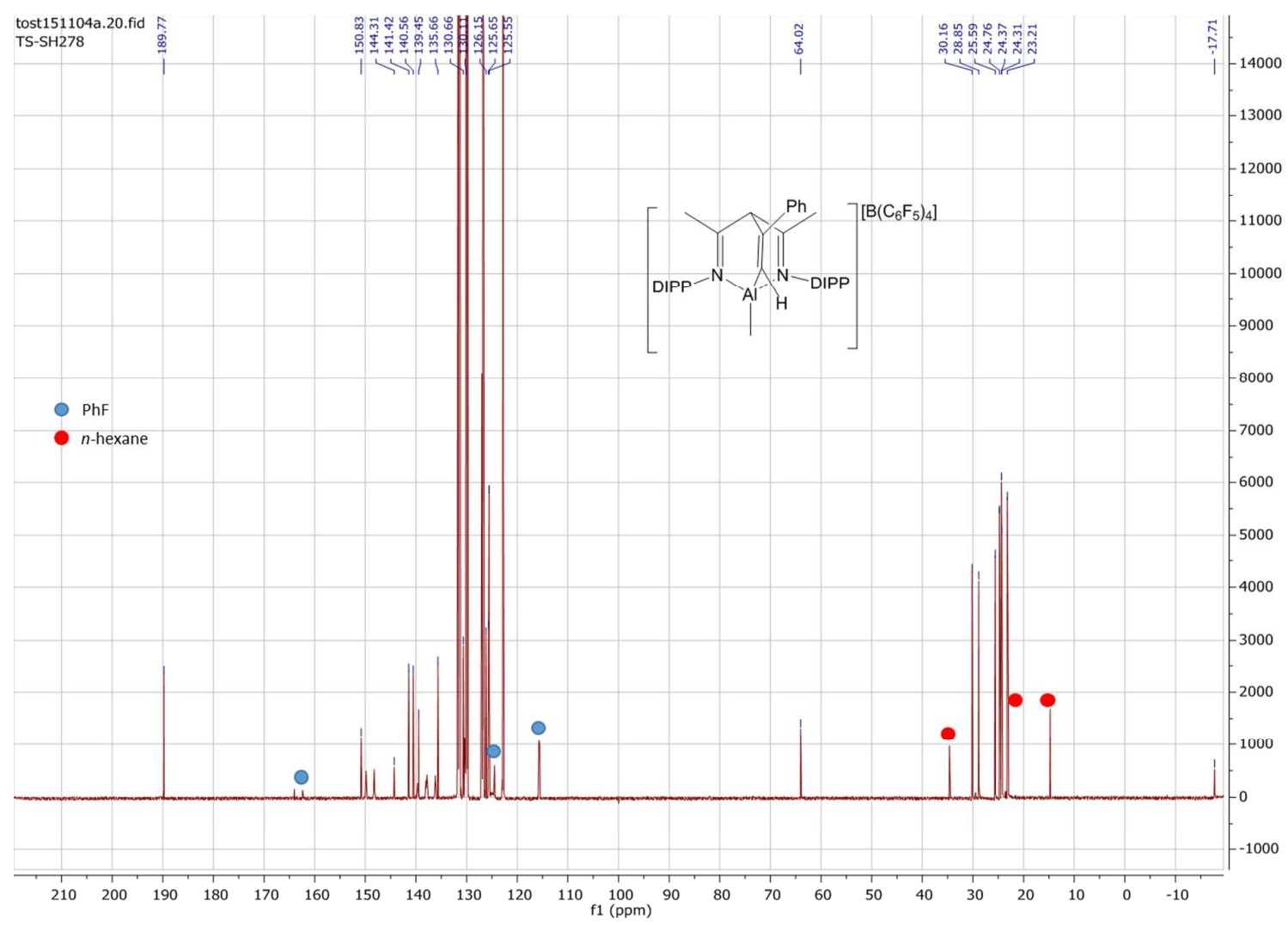


Figure S6 - Room temperature ${ }^{13} \mathrm{C}$ NMR spectrum of $1 / \mathrm{PPh}_{3}$ in $\mathrm{C}_{6} \mathrm{D}_{5} \mathrm{Br}$

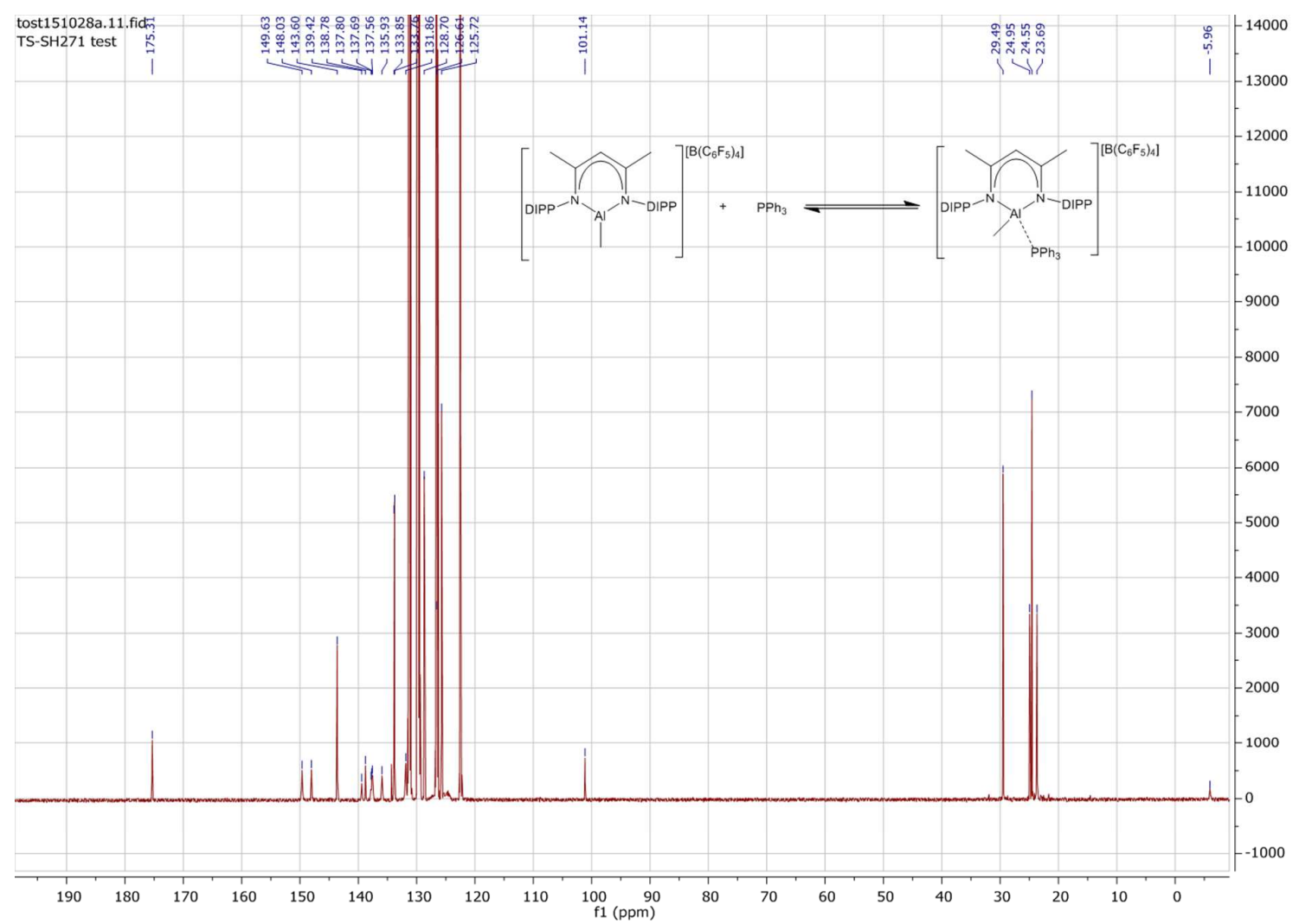

Figure S7 - Variable temperature ${ }^{1} \mathrm{H}$ NMR spectra of $1 / \mathrm{PPh}_{3}$

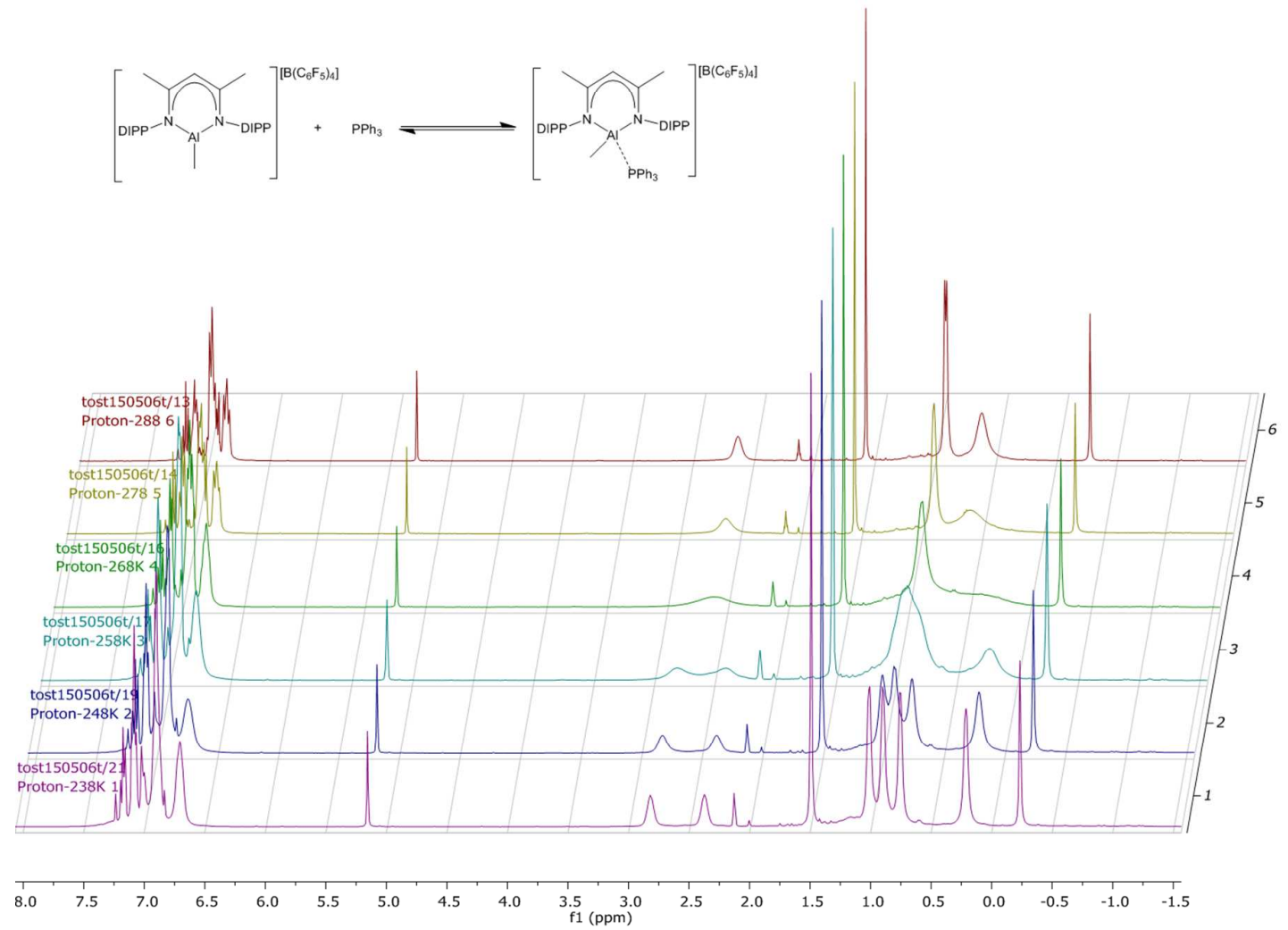


Figure $\mathrm{S} 8$-Variable temperature ${ }^{31} \mathrm{P}$ NMR spectrum of $1 / \mathrm{PPh}_{3}$

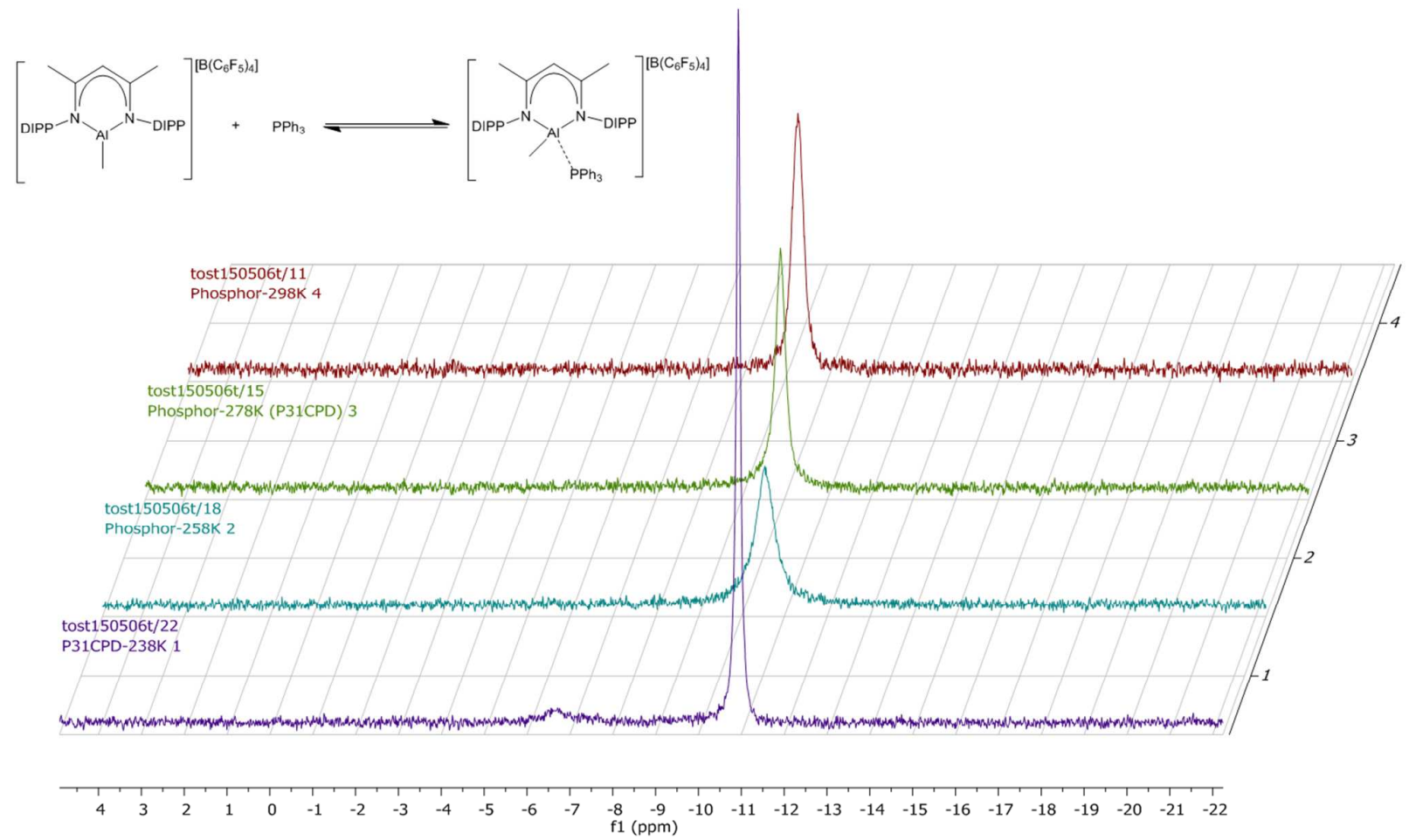

Figure S9 - Standard ${ }^{1} \mathrm{H}$ NMR spectrum (below) and selective TOCSY ${ }^{1} \mathrm{H}$ NMR spectrum (above) of compound $\mathbf{9}$, with irradiation of the triplet at $0.32 \mathrm{ppm}$.

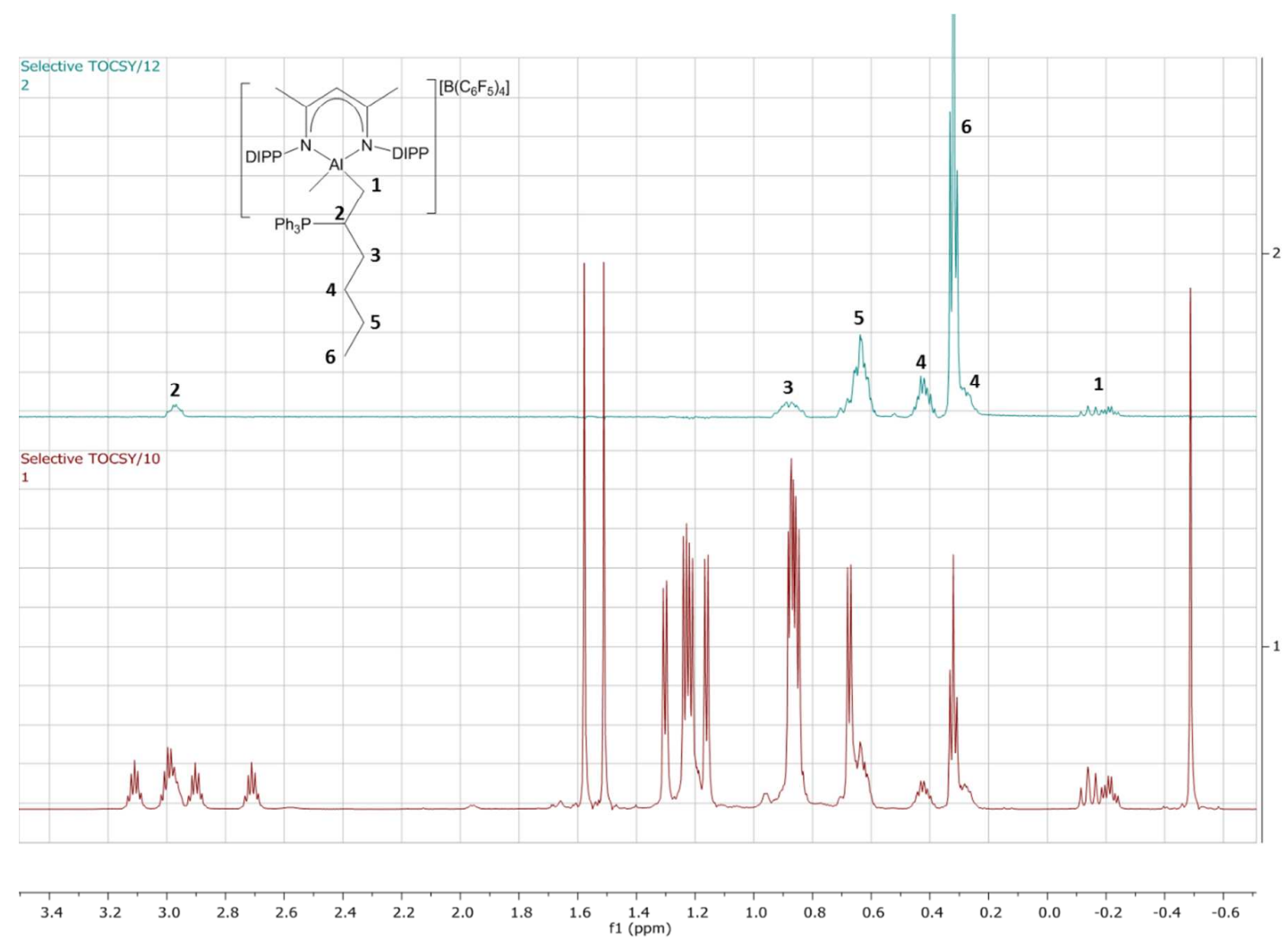


Figure $\mathrm{S} 10-{ }^{1} \mathrm{H}$ NMR spectrum of compound $\mathbf{1 0}$

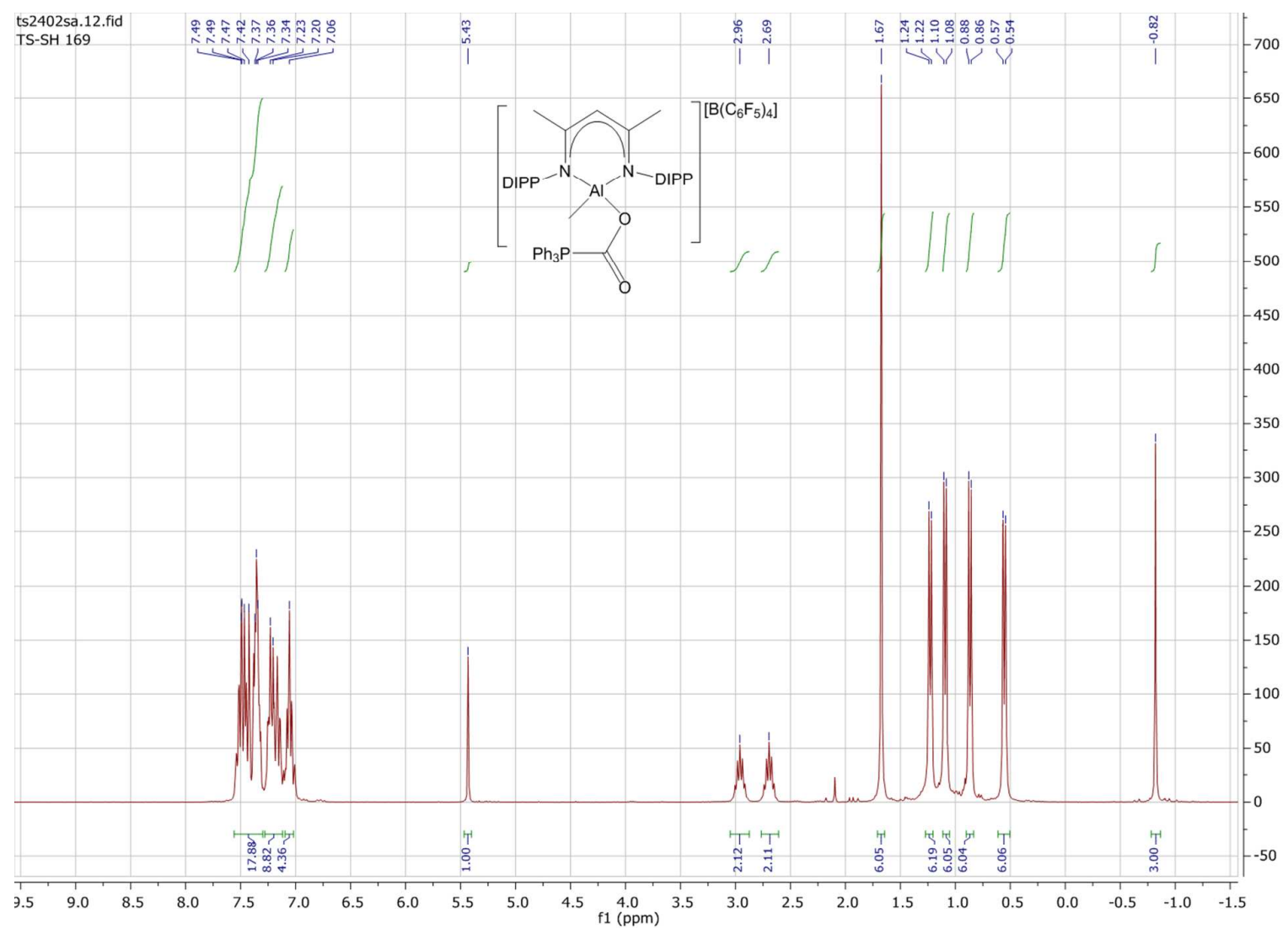

Figure $\mathrm{S} 11-{ }^{13} \mathrm{C}$ NMR spectrum of compound $\mathbf{1 0}$

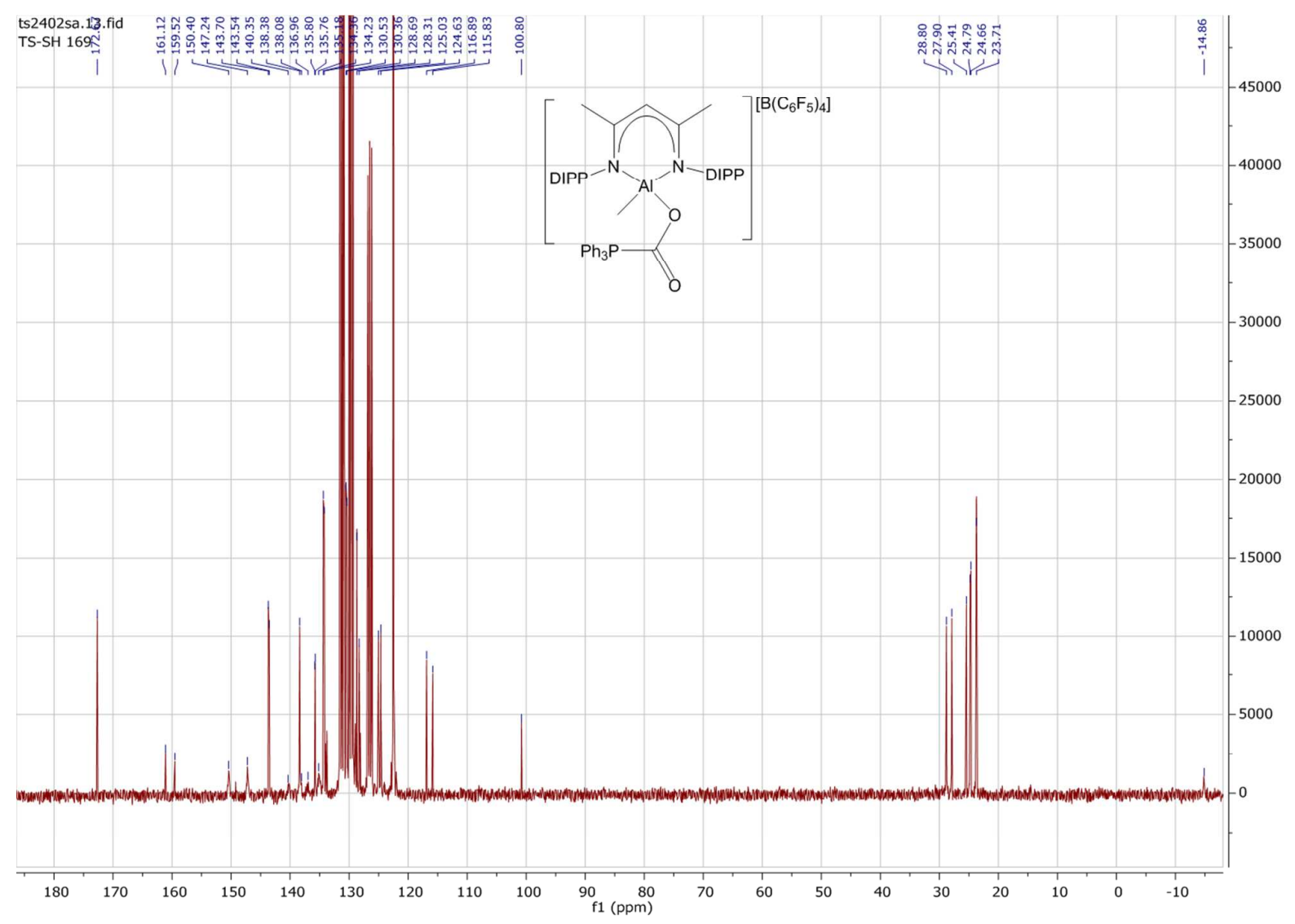


Figure S12 $-{ }^{31}$ P NMR spectrum of compound 10

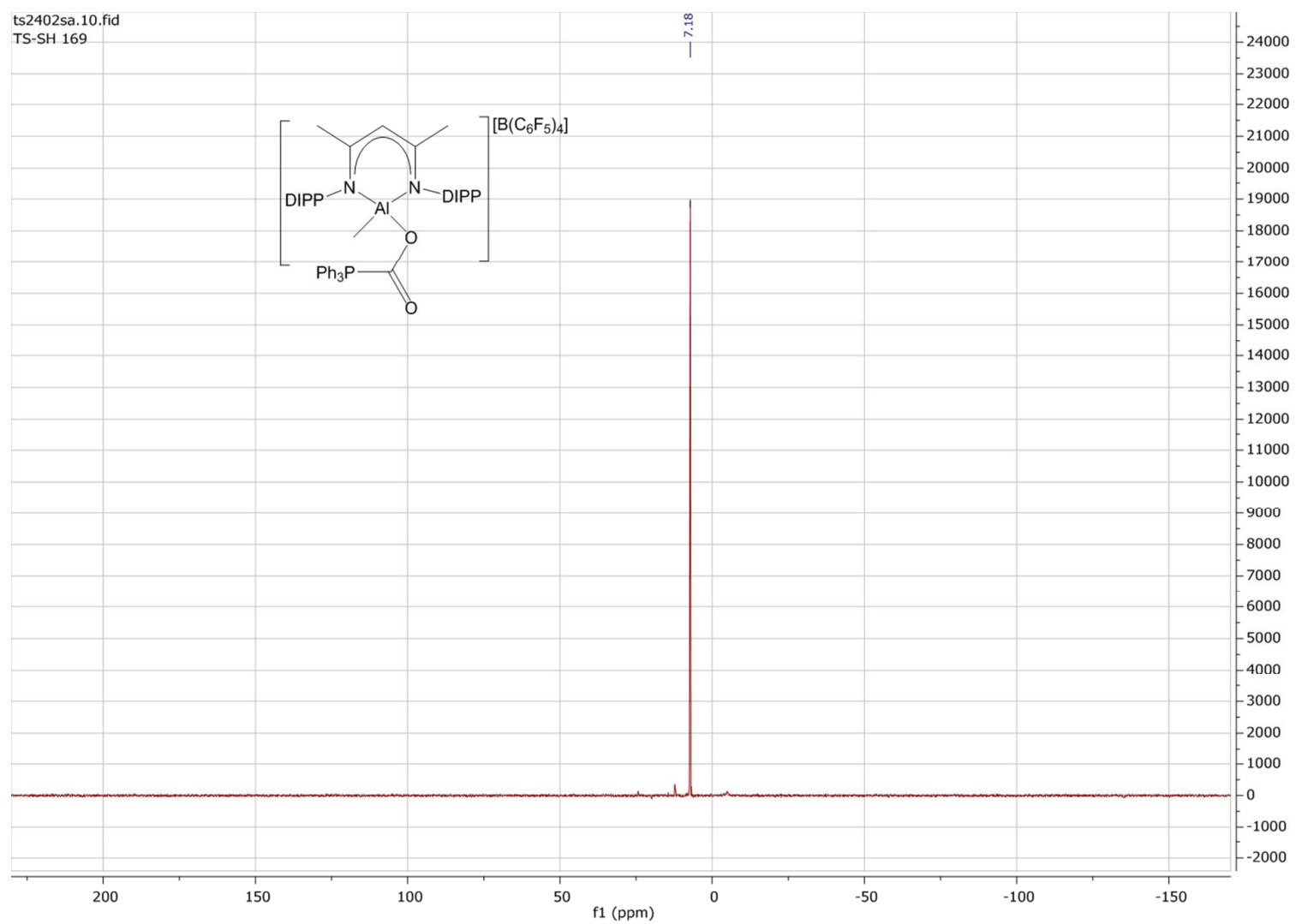

Figure $\mathrm{S} 13-{ }^{1} \mathrm{H}$ NMR spectrum of compound $\mathbf{1 1}$

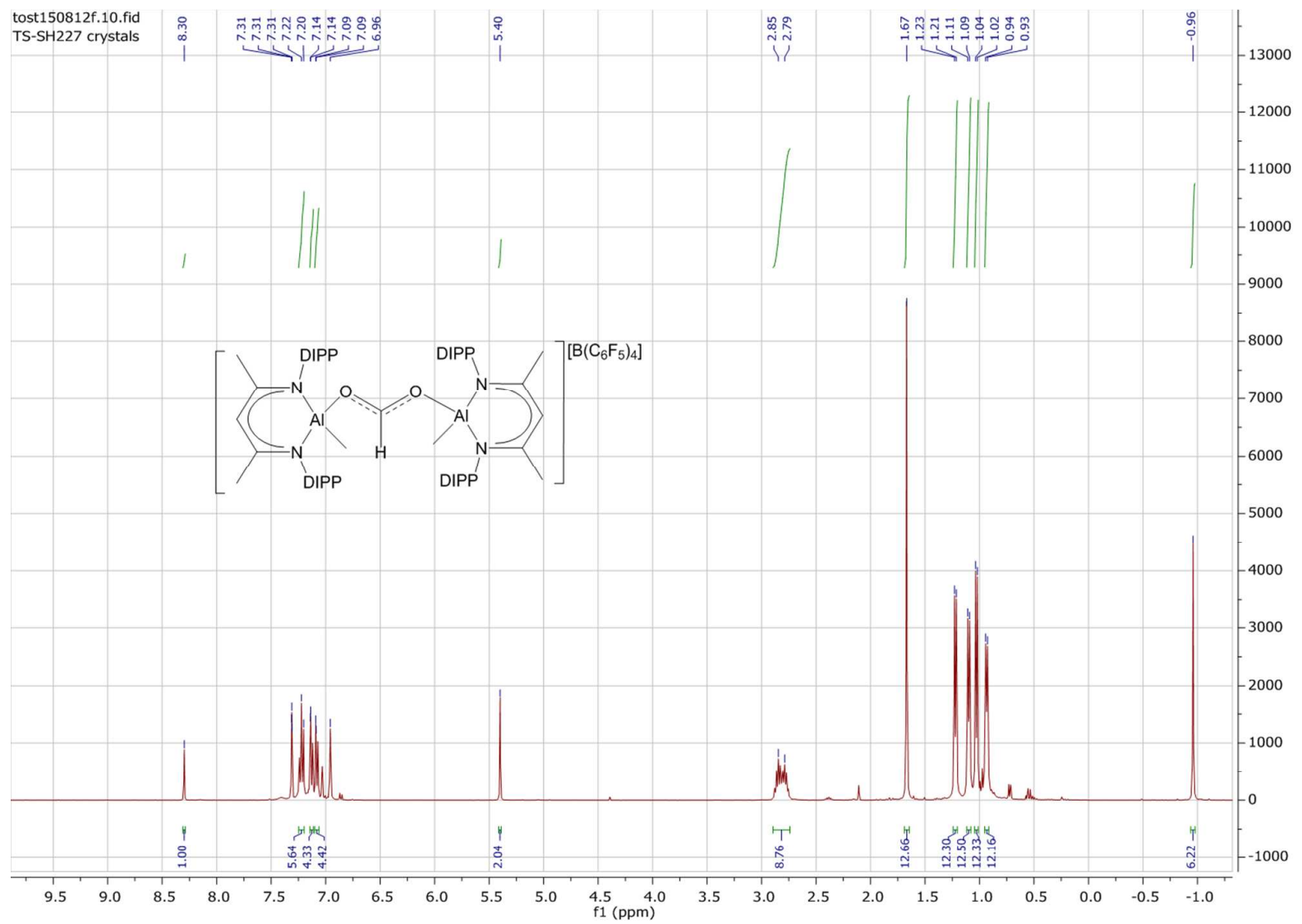


Figure S14 $-{ }^{13} \mathrm{C}$ NMR spectrum of compound $\mathbf{1 1}$

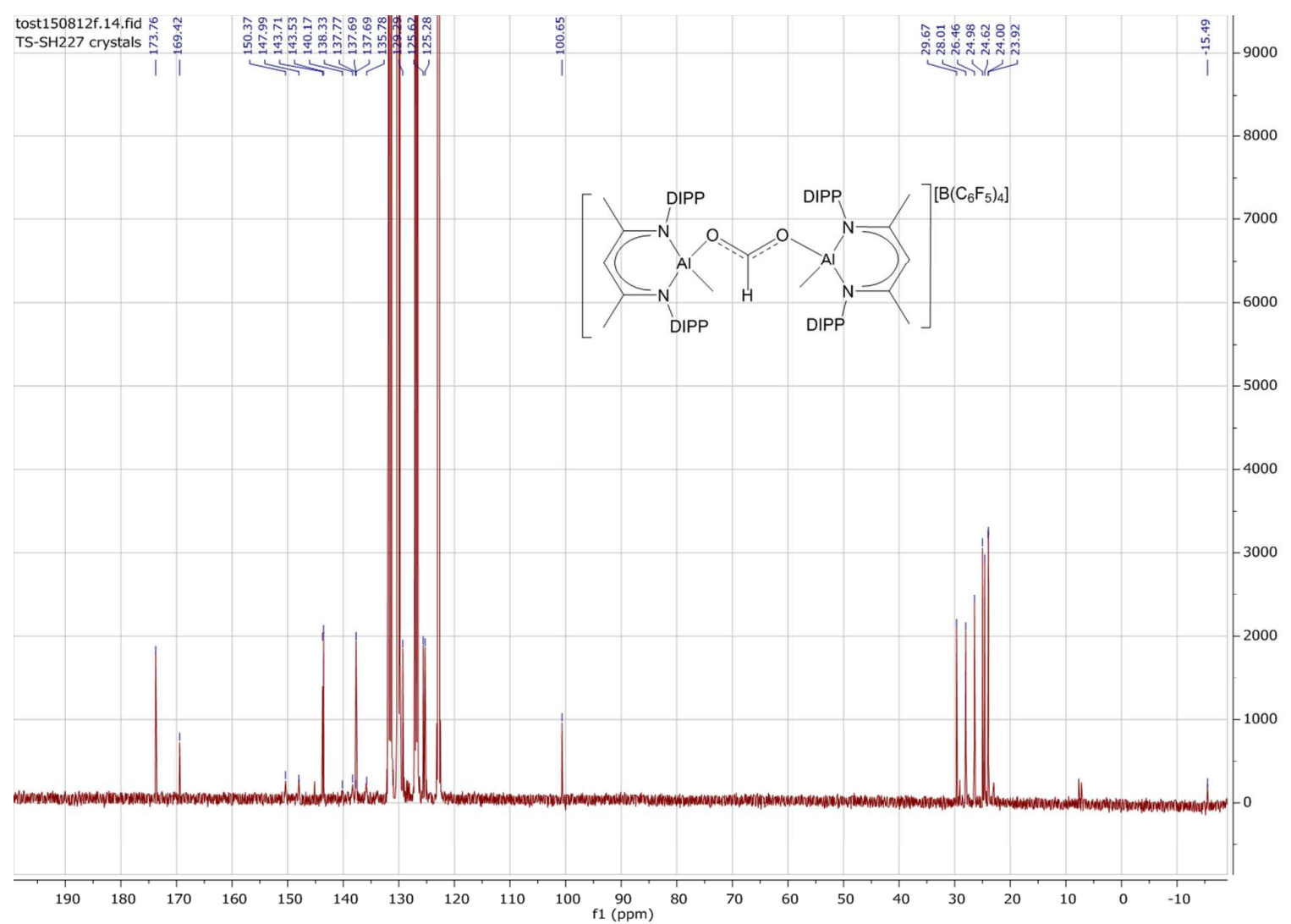

Figure S15 $-{ }^{1} \mathrm{H}$ NMR spectrum of compound 14

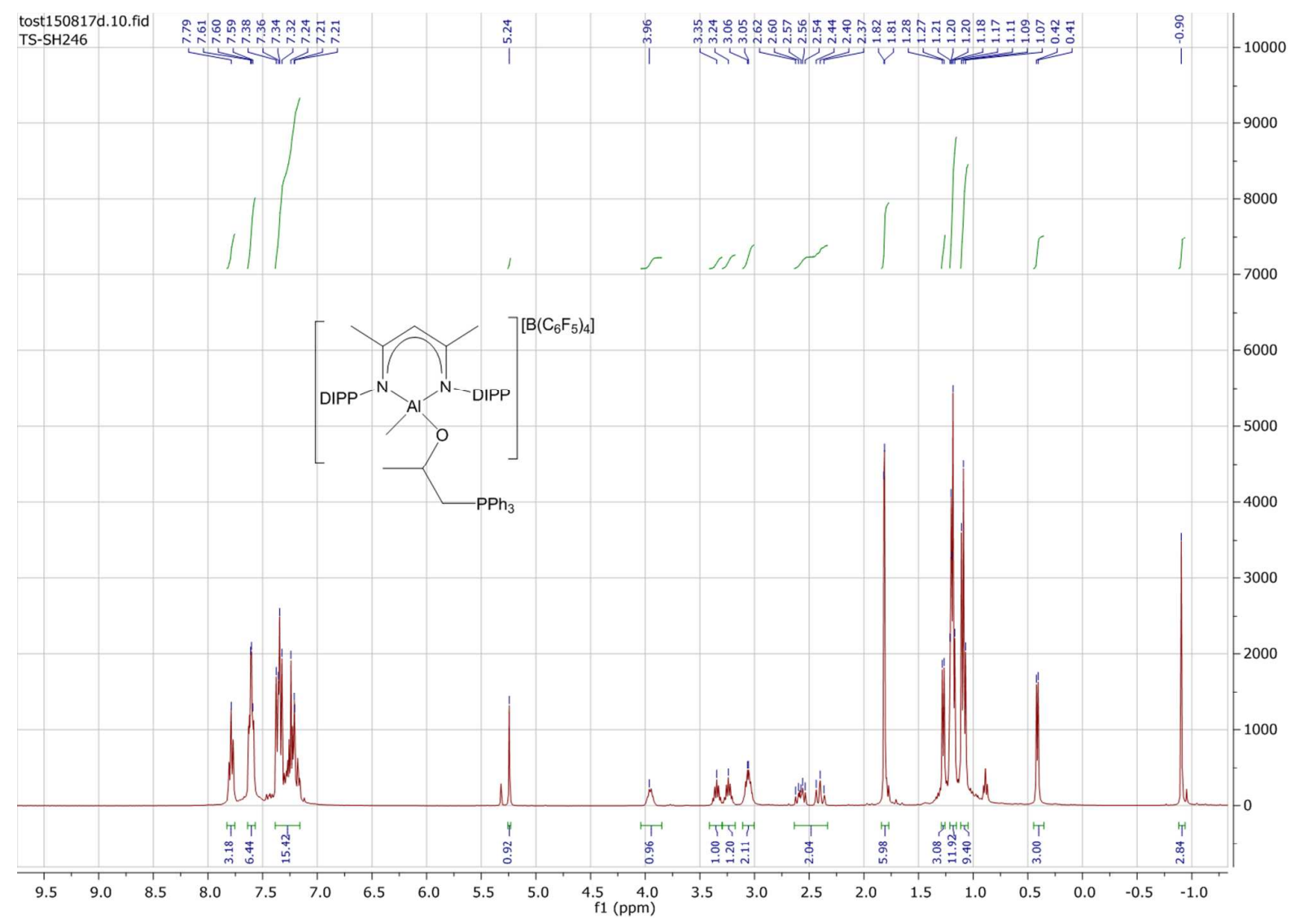


Figure S16 $-{ }^{13} \mathrm{C}$ NMR spectrum of compound 14

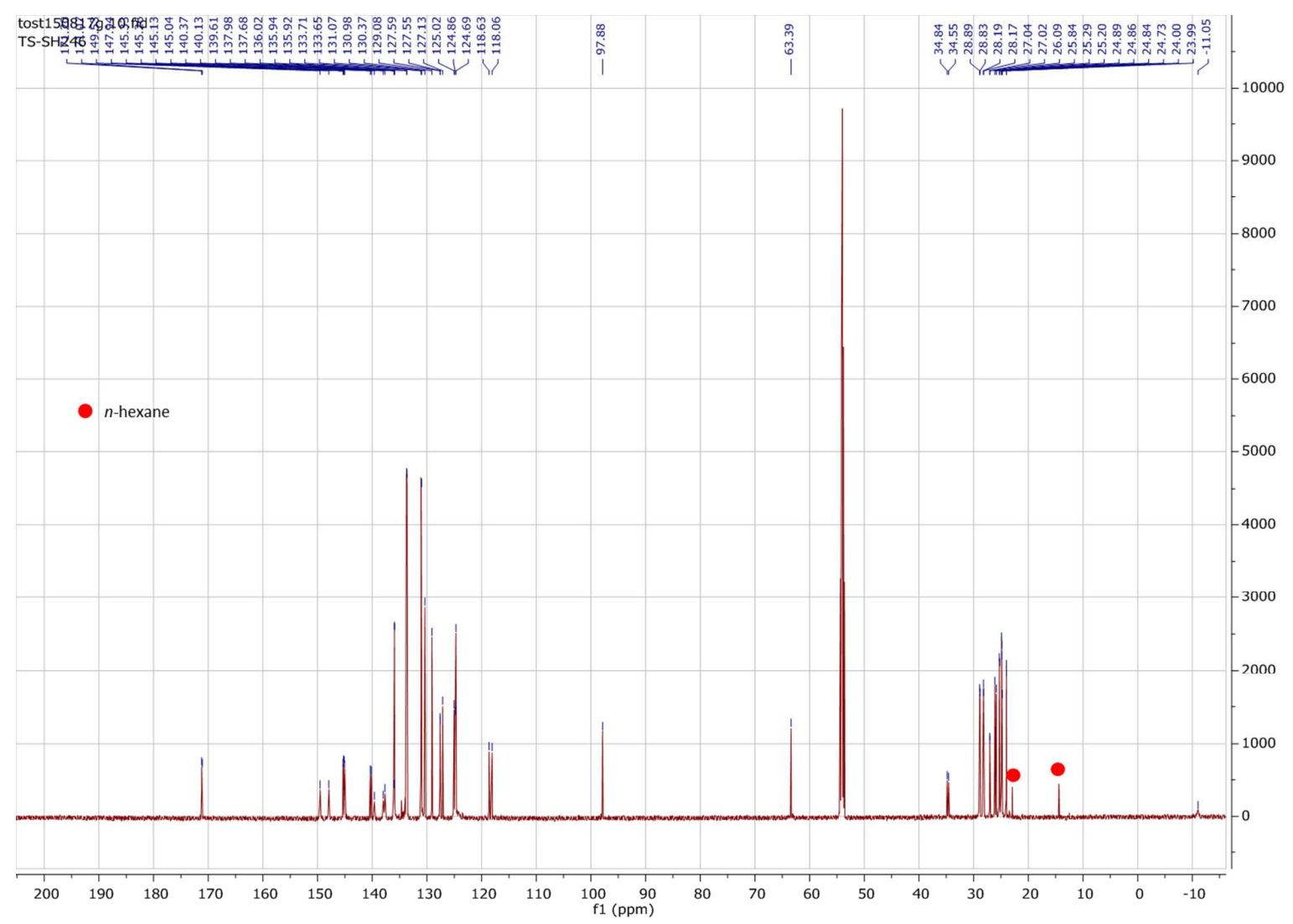

Figure S17 - ${ }^{31} \mathrm{P}$ NMR spectra for the Gutmann-Beckett Lewis acidity test

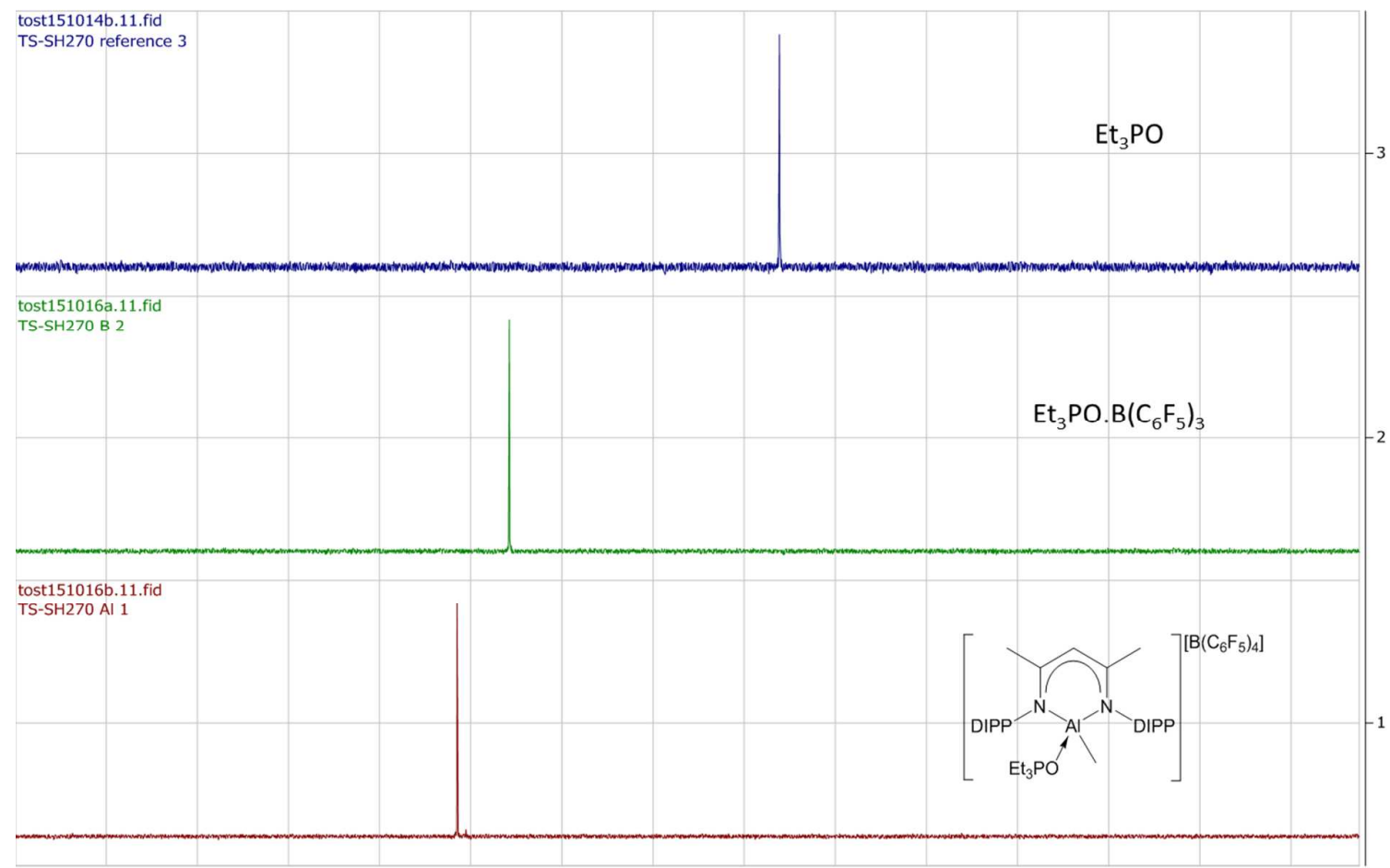

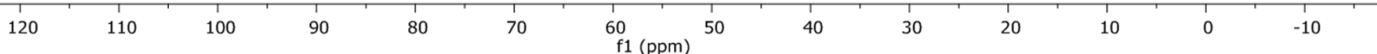

